\title{
Thecosomata e Gymnosomata (Mollusca, Gastropoda) da cadeia Fernando de Noronha, Brasil
}

\author{
Maria Eduarda de Larrazábal ${ }^{1} \&$ Valdeni Soares de Oliveira ${ }^{1}$
}

1 Departamento de Zoologia, Centro de Ciências Biológicas, Universidade Federal de Pernambuco. Avenida Professor Moraes Rego 1235, 50670-420 Recife, Pernambuco, Brasil.E-mail: mells@ufpe.br; valdeni.oliveira@bol.com.br

\begin{abstract}
Thecosomata and Gymnosomata (Mollusca, Gastropoda) from Fernando de Noronha chain, Brazil. The Mollusca Pteropoda from Fernando de Noronha Chain Northeastern Brazil were studied in order to know their taxonomy and distribution. Collections were carried out during the I Oceanographical Expedition of the REVIZEE Program (Live Resources of the Economical Exclusive Zone) from August to September 1995. The studied area is located between $03^{\circ} 04^{\prime} \mathrm{S}-04^{\circ} 23^{\prime} \mathrm{S}$ and $32^{\circ} 19^{\prime} \mathrm{W}-36^{\circ} 26^{\prime} \mathrm{W}$, comprising 35 stations. Sampling was performed through oblique hauls from $50 \mathrm{~m}$ depth to surface and from $100 \mathrm{~m}$ depth to surface, with a Bongo net (300 and $500 \mu \mathrm{m}$ mesh size, net diameter: $60 \mathrm{~cm}$ ), that was equipped with a flow meter. Samples in a total of 99 were preserved, immediately, in buffered $4 \%$ formaldehyde. Qualitative and quantitative analyses of the samples were performed based on total sample. The samples were analysed on a counting plate "Bogorov" type under a stereo microscope. A total of 8,258 organisms were identified belonging to: 8 families, 16 genera and 27 species. Limacina inflata (d'Orbigny, 1836) and Creseis virgula (Rang, 1828) were frequent and very frequent in the area outranking among the studied Pteropoda. The total density varied from 2.5 to 3,012.05 org. $\mathrm{m}^{-3}$. Highest richness was registered at the Continental Shelf and Slope with 1two species. The Pteropoda association showed higher differences between day and night collections. Limacina inflata caused this difference due to its higher density at night collections and it was independent of the depth, mesh size and temperature. Creseis virgula had its occurrence, mainly, during day time.
\end{abstract}

KEY WORDS. Brazil, Fernando de Noronha, Gymnosomata, Pteropoda, Thecosomata.

Existem aproximadamente 5.000 espécies de organismos zooplanctônicos holoplanctônicos descritas, representadas por diferentes grupos taxonômicos de invertebrados. Esses grupos são comumente encontrados no mar e formam frações significativas na comunidade planctônica (LaLli \& PARSONs 1993). Dentre eles destacam-se os Mollusca Thecosomata Blainville, 1824 e Gymnosomata Blainville, 1824, componentes do macrozooplâncton que compreende espécies com tamanho superior a 5 mm (Pérès 1976, Margaleff 1974, Ehrhardt \& Seguin 1978).

São conhecidos por "borboletas-marinhas", devido ao eixo da porção do pé ser modificado em duas nadadeiras (parapódios), estruturas semelhantes a asas, parecidas com aquelas das borboletas, tanto pela forma como pelo movimento. Essas "asas" são homólogas aos pés dos demais Gastropoda, possuindo as funções de natação, flutuação e alimentação (BÉ \& GILMER 1977, Gasca \& SuÁrez-Morales 1992, RupPert \& Barnes 1996).

Thecosomata possuem concha, freqüentemente com opérculo, parapódios geralmente expandidos para o nado, estômago químico guarnecido de quatro placas, mandíbulas laterais e uma rádula triseriada. Por sua abundância é o grupo mais conhecido entre os Pteropoda. A classificação dessa ordem está baseada na morfologia das conchas calcárias que contribui no ciclo de carbonato de cálcio $\left(\mathrm{CaO}_{3}\right)$ no oceano (Pruvot-Fol 1954, Boltovskoy 1981, van der Spoel \& Boltovskoy 1981, Ruppert $\&$ Barnes 1996, VAN DER SPoel 1996). Possuem tamanho pequeno, suas conchas são muitos frágeis e delgadas, quando externas (Euthecosomata), ou então, são flexíveis e gelatinosas quando internas (Pseudothecosomata), características adaptativas à vida planctônica. Thecosomata são herbívoros, possuindo uma forma de nutrição peculiar. Secretam uma rede de muco que se estende para capturar organismos fitoplanctônicos ou outras partículas alimentares, que são trazidas até à boca (BÉ \& GILMER 1977, Gasca \& Suárez-Morales 1992).

Gymnosomata são destituidos de concha, sem cavidade palial sendo a anatomia da parte mole do corpo a base para sua identificação. São seus caracteres mais importantes: os órgãos bucais e as brânquias. Possuem hábito carnívoro, são predadores ativos, principalmente dos Thecosomata. Predam suas presas perseguindo-as e capturando-as com órgãos complexos a exem- 
plo de ganchos, glândulas pegajosas e ventosas (Pruvot-FoL 1954, van der Spoel \& BoltovsKoy 1981, Ruppert \& Barnes 1996, VAN DER SPOel 1996). O hábito alimentar dos Gymnosomata foi estudado por LALLI (1970) em condições de laboratório.

Conhecidos desde o século XVII, os Pteropoda apresentam uma ampla distribuição oceânica, desde os pólos ao equador, da superfície até a zona batipelágica, sendo assim, de grande interesse para o conhecimento dos ecossistemas marinhos (LALLI \& Well Jr. 1973, van der Spoel \& Boltovskoy 1981, van der Spoel 1996).

A sua posição na cadeia trófica lhe confere uma importância ecológica, tanto como consumidores do micro e nanoplâncton, como alimento para espécies de peixes forrageiros. Em grandes concentrações, constituem uma parcela significativa na biomassa zooplanctônica (REsGalla \& MonTú 1994). Algumas espécies são consideradas como excelentes indicadores de massas d'água por possuírem limites de tolerância à temperatura e à salinidade muito definidos (CHEN \& Bé 1968, HAAGENSEN 1976, van der Spoel \& Boltovskoy 1981, Dadon 1984, Gasca \& Suárez-Moralles 1992, Resgalla \& Montú 1994).

Pteropoda do Atlântico Sul Ocidental têm sido pesquisados por vários autores, a exemplo de: BARTH (1968), BARTH \& Oleiro (1968), Magaldi (1974, 1981, 1983), Boltovskoy (1971), Rios $(1974,1985,1994)$, van der Spoel \& BoltovsKoy (1981), vaN Der Spoel \& Dadon (1999), Dadon (1982, 1984, 1990, 1992), Dadon \& Magaldi (1995), Dadon \& Chauvin (1998) e Resgalla \& Montú (1994). Apesar da existência de listagem de Pteropoda para o Atlântico Sul, apresentada em reuniões pertinentes ao zooplâncton, esses dados não constituem publicações que possam ser referidas. Para a região Nordeste do Brasil, informações a respeito da biologia, ecologia e biogeografia dos Pteropoda inexistem.

Com o objetivo de estudar a composição taxonômica e a distribuição dos Pteropoda coletados pelo REVIZEE NE I, da Cadeia Fernando de Noronha foi realizado o presente trabalho.

A cadeia Fernando de Noronha está representada pelo arquipélago de Fernando de Noronha $\left(03^{\circ} 30^{\prime} \mathrm{S}\right.$ e $\left.37^{\circ} 30^{\prime} \mathrm{W}\right)$, Atol das Rocas $\left(03^{\circ} 30^{\prime} \mathrm{S}\right.$ e $\left.32^{\circ} 30^{\prime} \mathrm{W}\right)$, e vários bancos submarinos como Grande $\left(03^{\circ} 50^{\prime} \mathrm{S}\right)$, Sírius $\left(04^{\circ} \mathrm{S}\right.$ e $\left.35^{\circ} 52^{\prime} \mathrm{W}\right)$, Guará $\left(03^{\circ} 55^{\prime} \mathrm{S}\right.$ e $\left.36^{\circ} 11^{\prime} \mathrm{W}\right)$ e o Drina $\left(03^{\circ} 50^{\prime} \mathrm{S}\right.$ e $\left.32^{\circ} 40^{\prime} \mathrm{W}\right)$, no extremo Oeste (Mabesoone \& Coutinho 1970).

Esta cadeia situa-se no sopé continental, composta por um segmento de montes, com direção Leste-Oeste, que se elevam de profundidades superiores a $4.000 \mathrm{~m}$ até à superfície. Estende-se do talude continental ao arquipélago de Fernando de Noronha que é o topo de um monte submarino, cuja base tem um diâmetro de aproximadamente $60 \mathrm{~km}$. Alguns montes caracterizam-se por apresentarem aplainados devido à erosão marinha, formando bancos tipos "guyots" (MABESOONE \& Coutinho 1970). O Atol das Rocas é um dos bancos da cadeia, com uma pequena parte de topo emersa; o banco Guará tem profundidade mínima em torno de $230 \mathrm{~m}$ e o Sírius tem seu topo entre 60 e $90 \mathrm{~m}$, sendo o mais raso deles (PALma 1984).

O arquipélago Fernando de Noronha é composto por 19 ilhas, que totalizam uma área de $26 \mathrm{~km}^{2}$, sendo as duas maiores ilhas: Rata e Fernando de Noronha. Fernando de Noronha é a principal ilha, com cerca de $18,4 \mathrm{~km}^{2}$, ocupando $91 \%$ da área total. Está situada a $345 \mathrm{~km}$ do Rio Grande do Norte entre os paralelos $3^{\circ} 00^{\prime}$ e $4^{\circ} 30^{\prime} \mathrm{S}$ e $32^{\circ} 00^{\prime}$ e $37^{\circ} 00^{\prime} \mathrm{W}$, estando-se na direção Leste-Oeste (MABEsoone \& Coutinho 1970).

O Atol das Rocas está situado entre as coordenadas $3^{\circ} 52^{\prime} \mathrm{S}$ e $33^{\circ} 49^{\prime} \mathrm{W}$, com superfície de 7,2 $\mathrm{km}^{2}$ e um diâmetro de 3,2 km. Encontra-se localizado a 144 milhas náuticas (cerca $260 \mathrm{~km}$ ) a Leste-Nordeste da costa do Estado do Rio Grande do Norte. A constituição do Atol difere dos demais por ser de origem calcária e não de origem coralínea (MABEsoone \& CouTinho 1970). Em relação ao sedimento, predominam restos de esqueletos de algas calcárias, carapaças de foraminíferos, fragmentos de conchas de moluscos, ossos de aves e peixes, como também as excretas das aves (guano) (Mabesoone \& CoutinHo 1970).

\section{MATERIAL E MÉTODOS}

O material estudado, foi coletado entre os meses de agosto e setembro de 1995, pelo Navio Oceanográfico Antares da DHN/Marinha do Brasil, parte integrante das atividades do Programa "Avaliação do Potencial Sustentável de Recursos Vivos da Zona Econômica Exclusiva" (REVIZEE - NORDESTE I), entre os paralelos $03^{\circ} 04^{\prime} \mathrm{S}$ e $04^{\circ} 23^{\prime} \mathrm{S}$ a $32^{\circ} 19^{\prime} \mathrm{W}$ e $36^{\circ} 26^{\prime} \mathrm{W}$. Os exemplares encontram-se depositados no acervo do Laboratório de Biologia da Conservação, Departamento de Zoologia, Centro de Ciências Biológicas da Universidade Federal de Pernambuco.

Foram estudadas 35 estações, totalizando 99 amostras. A coluna d'água foi amostrada através de rede do tipo bongo acoplada de fluxômetro, com aros de $60 \mathrm{~cm}$ de diâmetro e malhas coletoras de 300 e $500 \mu \mathrm{m}$, através de dois arrastos oblíquos por estação: um de 0-50 m de profundidade e outro de 0-100 m com duração de 10 minutos cada, sendo a velocidade do navio de dois nós. As amostras foram acondicionadas em recipientes plásticos e fixadas em formaldeído a $4 \%$, para posterior análise em laboratório.

Em laboratório, o material foi lavado, colocado em placa do tipo "Bogorov" e observado sob estereomiscroscópio binocular. De cada amostra foi retirada a totalidade dos Pteropoda da fauna acompanhante, com o auxílio de conta-gotas e pincel n. ${ }^{\circ}$ 00. Os espécimens foram acondicionados em recipientes de acrílico de $10 \mathrm{ml}$ de volume, sendo fixados em álcool a $70^{\circ} \mathrm{GL}$ glicerinado a 10\%, e identificados segundo: PRUvOT-FoL (1942), Tesch (1946a a b), van der Spoel $(1967,1972)$, Авbott (1974), BoLtovskoy (1981), van der Spoel \& Boltovskoy (1981), van der SPoel $\&$ Dadon (1999), entre outros. De acordo com van der Spoel $(1967,1972)$ foi confecionada a sinopse dos Pteropoda coligidos em Fernando de Noronha.

Os dados referentes à Oceanografia abiótica (salinidade, temperatura, oxigênio dissolvido, potencial hidrogeniônico) foram coletados pelo Laboratório de Oceanografia Química, Universidade Federal de Pernambuco, servindo às análises estatísticas.

A Freqüência de Ocorrência (\%) foi calculada através da expressão $\mathrm{F}=\mathrm{a} \times 100 / \mathrm{A}$, onde $a$ é o número de amostras contendo a espécie e $A$ o número total de amostras obtidas. A partir dos resultados foi possível distinguir as seguintes categorias: F > 70\% - Muito freqüente; $40 \%<\mathrm{F} \leq 70 \%$ - Freqüente; $10 \%<\mathrm{F} \leq 40 \%$ - Pouco freqüente e, F $\leq 10 \%$ - Esporádica. Para a obtenção da Abundância Relativa (\%) dos Pteropoda evidenciados, foi utilizada a fórmula $\mathrm{Ar}=\mathrm{N} \times 100 / \mathrm{Na}$, onde: $\mathrm{N}$ é o número total de organismo de cada táxon na amostra e $\mathrm{Na}$ o número total de organismo na amostra. Os organismos foram classificados nas categorias: Ar $>70 \%$ - Dominante; $40 \% \geq \mathrm{Ar}>70$ - Abundante; $10 \%>\mathrm{Ar} \geq 40 \%$ - Pouco Abundante e, $\mathrm{Ar} \leq 10 \%$ - Raro. A Densidade (org. $\mathrm{m}^{-3}$ ) foi calculada a partir da expressão $\mathrm{N}^{\circ} \operatorname{org} \times \mathrm{m}^{-3}=\mathrm{n} / \mathrm{v}$, onde $\mathrm{n}$ é o número de organismos na amostra, v é o volume de água filtrada por 
Tabela I. Sinopse dos Pteropoda (Mollusca, Gastropoda) identificados na Cadeia Fernando de Noronha, durante a I Campanha do REVIZEE/NE, em 1995.

\begin{tabular}{|c|c|c|}
\hline Subordem & Família & Espécie \\
\hline \multirow[t]{12}{*}{ Euthecosomata Meisenheimer, 1905} & Limacinidae Gray, 1847 & Limacina helicina (Phipps, 1774) \\
\hline & & Limacina bulimoides (d'Orbigny, 1836) \\
\hline & & Limacina trochiformis (d'Orbigny, 1836) \\
\hline & & Limacina inflata (d'Orbigny, 1836) \\
\hline & Cavoliniidae Fischer, 1883 & Cavolinia inflexa (Lesueur, 1813) \\
\hline & & Diacria trispinosa (Blainville, 1821) \\
\hline & & Clio pyramidata Linnaeus, 1767 \\
\hline & & Clio sp. \\
\hline & & Creseis acicula (Rang, 1828) \\
\hline & & Creseis virgula (Rang, 1828) \\
\hline & & Styliola subula (Quoy and Gaimard, 1827) \\
\hline & & Cuvierina columnella (Rang, 1827) \\
\hline Pseudothecosomata Meisenheimer, 1905 & Cymbuliidae Cantraine, 1841 & Corolla cupula Rampal, 1996 \\
\hline \multirow{10}{*}{$\begin{array}{l}\text { Gymnosomata Blainville, } 1824 \\
\text { (sensu van der Spoel 1976) }\end{array}$} & Clionidae (Phipps, 1774) & Clione limacina (Phipps, 1774) \\
\hline & & Clione sp. \\
\hline & Cliopsidae Dall, 1889 & Cliopsis sp. \\
\hline & & Cliopsis aff. \\
\hline & Notobranchaeidae Pelseneer, 1886 & Notobranchaea tetrabranchiata (Bonnevie, 1913) \\
\hline & $\begin{array}{l}\text { Pneumodermatidae Pelseneer, } 1887 \text { ex } \\
\text { Latreille, } 1825\end{array}$ & Pneumoderma sp. \\
\hline & & Pneumodermopsis macrochira Meisenheirmer,1905 \\
\hline & & Pneumodermopsis paucidens (Boas, 1886) \\
\hline & & Spongiobranchaea australis d'Orbigny, 1836 \\
\hline & & Spongiobranchaea sp. \\
\hline
\end{tabular}

metro cúbico $\left(\mathrm{m}^{3}\right)$ durante o tempo de arrasto, sendo os resultados multiplicados por $10^{3}$ para melhor visualização gráfica.Para a análise multivariada foram comparados: malhas coletoras (300 e $500 \mu \mathrm{m})$, estratos $(0-50$ e $0-100 \mathrm{~m})$, turnos (dia e noite) e temperatura (faixas de $17-19^{\circ} \mathrm{C} ; 20-25^{\circ} \mathrm{C} ;>25^{\circ} \mathrm{C}$ ), utilizando as densidades. Foi aplicada a Análise Multivariada de Similaridade (ANOSIM), onde de acordo com Clarke \& W ARWICK (1994) os valores < 5\% são considerados significativos e aqueles $>5 \%$ não significativos.

Aplicou-se ainda a análise de ordenação do tipo Multi Dimensional Scale (MDS) objetivando-se evidenciar a estrutura dos conjuntos de dados (Clarke \& WarWick 1994). Após utilizar a MDS avaliou-se o stress de cada uma ordenação sendo valores $<0,2 \%$ considerados significativos.

\section{RESULTADOS}

Para a área da Cadeia Fernando de Noronha foram identificados 8.258 Pteropoda, distribuídos em sete famílias,
16 gêneros e 27 espécies (Tab. I).

\section{Malha coletora $300 \mu \mathrm{m}$}

Quanto à freqüência de ocorrência em ambos os estratos (0-50 e 0-100 $\mathrm{m}$ ) as espécies que mais se destacaram foram: Limacina inflata (d'Orbigny, 1836), Creseis virgula (Rang, 1828), Cavolinia inflexa (Lesueur, 1813), Cuvierina columnella (Rang, 1827) e Limacina bulimoides (d'Orbigny, 1836). As demais espécies foram inseridas nas categorias pouco freqüentes e raras (Fig. 1A).

Em se tratando da abundância relativa, no estrato de 0$50 \mathrm{~m}$, Limacina inflata e Creseis virgula foram dominantes na maioria das estações. Cuvierina columnella foi dominante nas estações 48, 51 e 59, seguida por Cavolinia inflexa, Creseis virgula e Hyalocylis striata (Rang, 1828) (Fig. 2A). No estrato 0-100 m, as espécies dominantes foram Limacina inflata e Creseis virgula, seguidas por Limacina trochiformis (d’Orbigny, 1836) e Cavolinia inflexa (Fig. 3A).

A densidade (org. $\mathrm{m}^{-3}$ ) das espécies de Pteropoda na Cadeia

Revista Brasileira de Zoologia 20 (2): 351-360, junho 2003 

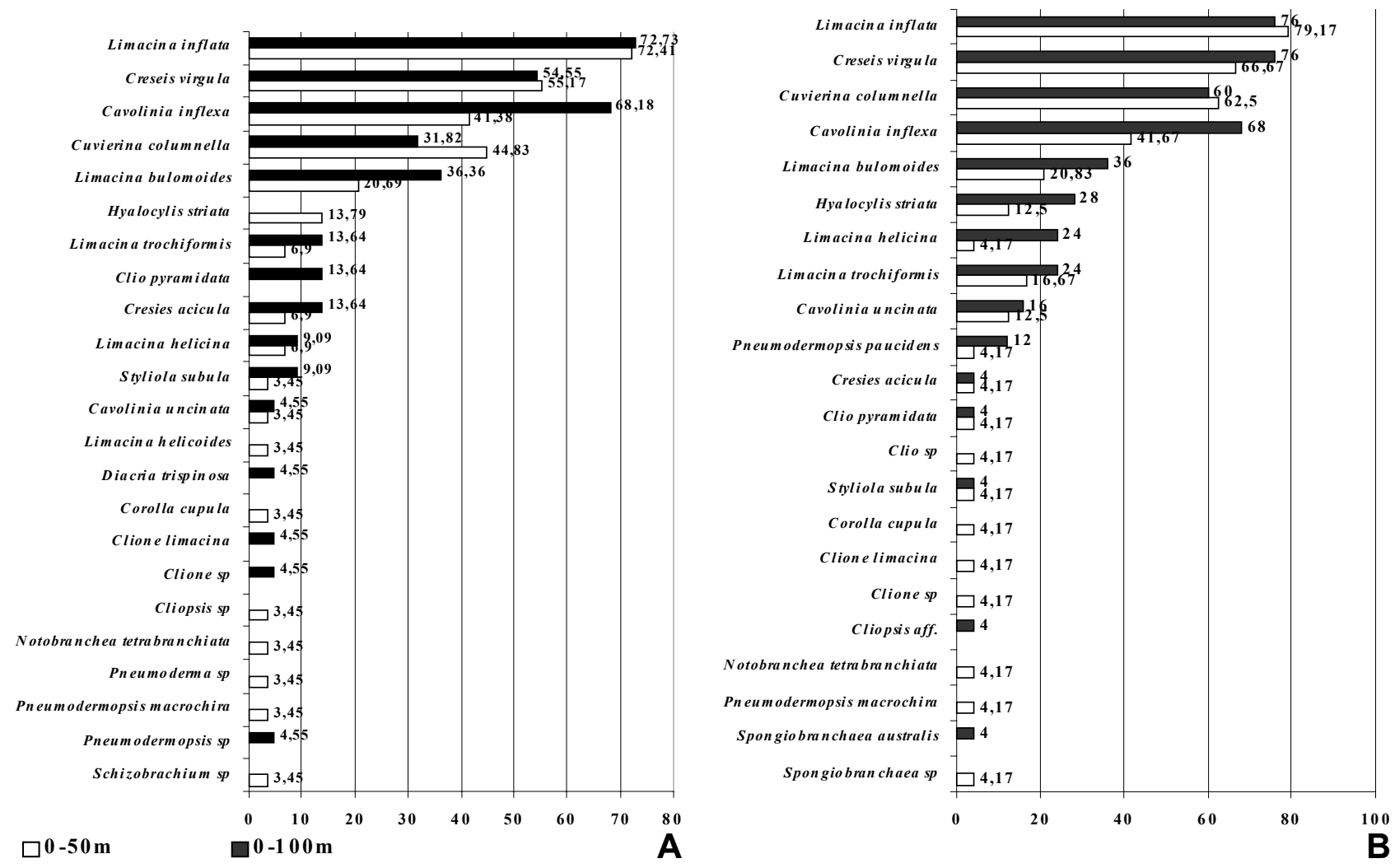

Figura 1. Freqüência de ocorrência dos Pteropoda coletados na Cadeia Fernando de Noronha, com malha de (A) $300 \mu \mathrm{m}$ e (B) $500 \mu \mathrm{m}$ nos estratos de 0-50 e 0-100 m, durante a I Campanha do REVIZEE/NE, em 1995.

Fernando de Noronha, variou conforme as estações, profundidades e horários de coleta. Para as amostras do estrato de 0-50 m a densidade variou de 3,32 org. $\mathrm{m}^{-3}$, na estação 70 em coleta diurna (10h e $21 \mathrm{~min}$ ) a $3.012,05$ org. $\mathrm{m}^{-3}$, na estação 44 , em coleta noturna ( $22 \mathrm{~h}$ e $51 \mathrm{~min}$ ). No estrato de $0-100 \mathrm{~m}$, a densidade variou de 2,5 org. $\mathrm{m}^{-3}$, na estação 80 em coleta diurna ( $7 \mathrm{~h}$ e $46 \mathrm{~min})$, a $2.802,08 \mathrm{org} \cdot \mathrm{m}^{-3}$ na estação $61 \mathrm{em}$ coleta noturna (3h e 50min) (Fig. 4A).

\section{Malha coletora $500 \mu \mathrm{m}$}

Quanto à freqüência de ocorrência as espécies que mais se destacaram em ambos os estratos $(0-50$ e 0-100 m) foram: Limacian inflata, Creseis virgula, Cavolinia inflexa e Cuvierina columnella. As demais espécies foram inseridas nas categorias pouco freqüentes e raras (Fig. 1B).

No estrato de $0-50 \mathrm{~m}$ Limacina inflata, Cuvierina columnella, Creseis virgula, Creseis acicula (Rang, 1828) e Cavolinia inflexa foram as mais abundantes (Fig. 2B). No estrato de 0-100 $\mathrm{m}$ foram Limacina inflata, Cavolinia inflexa, Creseis virgula, Limacina tochiformis (Fig. 3B).

A densidade no estrato de $0-50 \mathrm{~m}$ variou de 3,32 org. $\mathrm{m}^{-3}$, na estação 102 em coleta diurna (9h e 10min) a 1.937,93 org.m 3 , na estação 61 em coleta noturna ( 3 h e $26 \mathrm{~min}$ ). No estrato de $0-100 \mathrm{~m}$ a densidade variou de 4,05 org. ${ }^{-3}$, na estação 47 em coleta diurna (7 h e $00 \mathrm{~min}$ ) a $2.208,41 \mathrm{org} \cdot \mathrm{m}^{-3}$, na estação 61 em coleta noturna (3h e 50min) (Fig. 4B).

Quando a ANOSIM foi aplicada à densidade (org. $\mathrm{m}^{-3}$ ) resultou em diferenças não significativas para a Cadeia Fernando de Noronha, levando-se em consideração os seguintes aspectos: 1 ) entre as malhas coletoras $(\mathrm{r}=8,5 \%) ; 2$ ) na malha coletora de $300 \mu \mathrm{m}$ entre os estratos $0-50$ e $0-100 \mathrm{~m}(\mathrm{r}=46,7 \%) ; 3)$ na malha coletora de $500 \mu \mathrm{m}$ entre os estratos $0-50$ e $0-100 \mathrm{~m}(\mathrm{r}=$ $81,1 \%) ; 4$ ) na malha coletora de $300 \mu \mathrm{m}$ no estrato $0-50 \mathrm{~m}$ entre os turnos (diurno e noturno) $(\mathrm{r}=5,6 \%) ; 5)$ malha coletora de $300 \mu \mathrm{m}$ no estrato $0-100 \mathrm{~m}$ entre os turnos (diurno e noturno) $(\mathrm{r}=0,1)$; 6) na malha coletora de $500 \mu \mathrm{m}$, no estrato $0-50 \mathrm{~m}$ entre os turnos (diurno e noturno) $(\mathrm{r}=5 \%) ; 7$ ) entre os estratos $0-50$ e $0-100 \mathrm{~m}$, independente das malhas coletoras, turnos e temperatura $(\mathrm{r}=5 \%) ; 8)$ entre as temperaturas, independente das malhas coletoras, turnos e temperatura $(\mathrm{r}=84,8 \%)$.

Apresentaram diferenças significativas os seguintes aspectos: 1) na malha coletora de $300 \mu \mathrm{m}$ no estrato $0-100 \mathrm{~m}$ entre os turnos (diurno e noturno) $(\mathrm{r}=0,1 \%)$. Levando-se em consideração os percentuais das espécies responsáveis por estas diferenças, Limacina inflata, Creseis virgula, Limacina bulimoides, Cuvierina columnella e Cavolinia inflexa apresentaram maiores densidades e abundância no período noturno; 2) na malha 


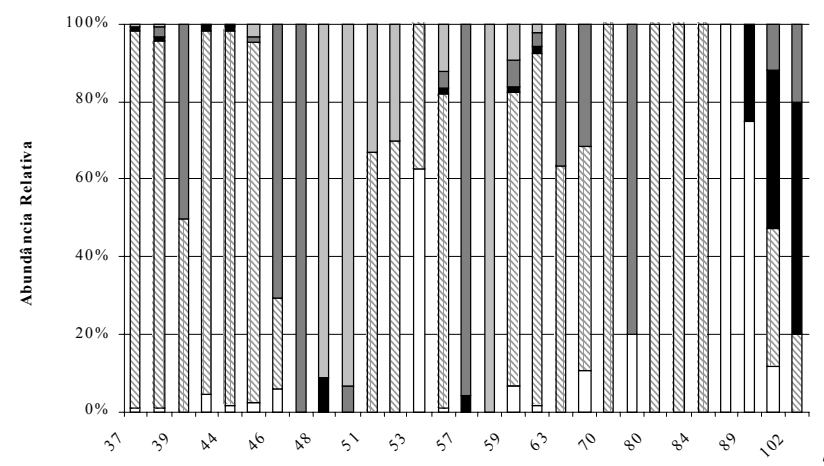

Esta ções

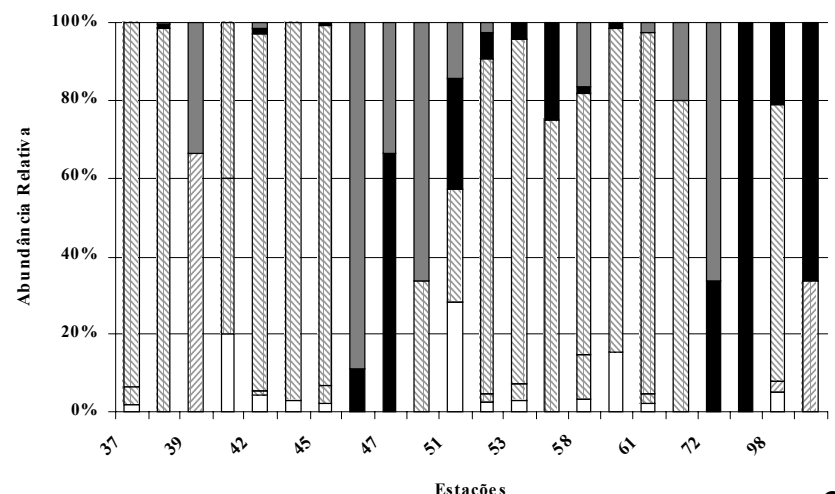

Limacina bulimoides

$3 A$

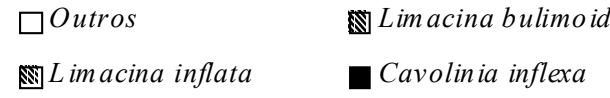

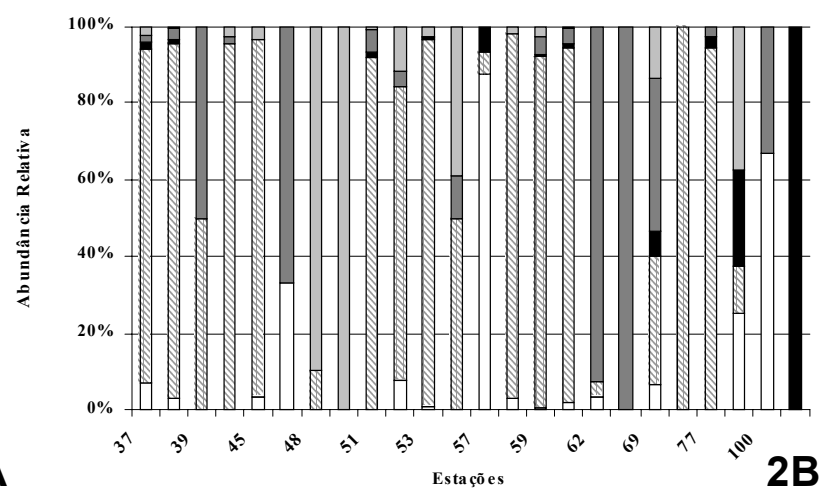

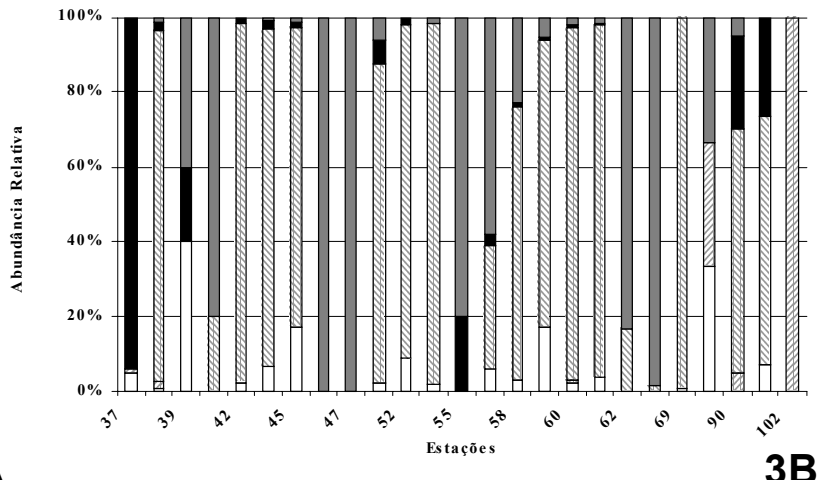

Figuras 2-3. Abundância relativa dos Pteropoda coletados na Cadeia Fernando de Noronha, com malha de (2A) $300 \mu \mathrm{m}$ e (2B) $500 \mu \mathrm{m}$ no estrato de 0-50 m; com malha de (3A) $300 \mu \mathrm{m}$ e (3B) $500 \mu \mathrm{m}$ no estrato de 0-100 m, durante a I Campanha do REVIZEE/ NE, em 1995.

coletora de $300 \mu \mathrm{m}$ independente dos estratos entre os turnos $(\mathrm{r}=0,1 \%)$. Levando-se em consideração os percentuais das espécies responsáveis por estas diferenças em ordem decrescente Limacina inflata apresentou maior densidade e abundância no horário noturno, Creseis virgula no horário diurno e Cuvierina columnella, Cavolinia inflexa e Limacina bulimoides no período noturno; 3) na malha coletora de $500 \mu \mathrm{m}$ no estrato $0-100 \mathrm{~m}$ entre os turnos (diurno e noturno) $(\mathrm{r}=0,1 \%)$. Levando-se em consideração os percentuais das espécies responsáveis por estas diferenças em ordem decrescente, Limacina inflata apresentou maior densidade e abundância no período noturno, Creseis virgula no período diurno, Cavolinia inflexa, Cuvierina columnella e Limacina bulimoides no período noturno; 4) na malha coletora de $500 \mu \mathrm{m}$ entre os turnos (diurno e noturno) $(\mathrm{r}=0,1 \%)$. Levando-se em consideração os percentuais das espécies responsáveis por estas diferenças em ordem decrescente, Limacina inflata apresentou maior densidade e abundância no período noturno, Creseis virgula no período diurno, Cuvierina columnella e Cavolinia inflexa no período noturno; 5) entre os turnos, independente das malhas coletoras, estrato e temperatura $(r=0,3 \%)$. Levandose em consideração os percentuais das espécies responsáveis por estas diferenças em ordem decrescente, Limacina inflata e
Cuvierina columnella apresentaram maiores densidades e abundância no período noturno, Creseis virgula no período diurno, Cavolinia inflexa e Limacina bulimoides no período noturno.

Na MDS é possível identificar a formação dos grupos A e $\mathrm{B}$, sendo o primeiro formado pelas amostras do turno noturno e o segundo pelo turno diurno. No mesmo gráfico, observa-se ainda que o grupo A apresenta-se mais compacto do que o grupo $\mathrm{B}$, revelando a grande similaridade entre as amostras do primeiro grupo, o que não se repete no segundo (Fig. 5). As espécies responsáveis pela separação entre os grupos foram basicamente Limacina inflata, L. bulimoides, Cavolinia inflexa e Cuvierina columnella, sendo mais abundantes nas amostras noturnas. Creseis virgula foi mais abundante nas amostras diurnas.

\section{DISCUSSÃO}

Os Pteropoda têm grande importância ecológica no que diz respeito a sua posição na cadeia trófica, que em grandes concentrações, constituem uma parcela significativa na biomassa zooplanctônica. Dentro do zooplâncton são pouco estudados nas zonas tropicais (VAN DER SPOel \& BoltovsKoy 1981). A Cadeia 

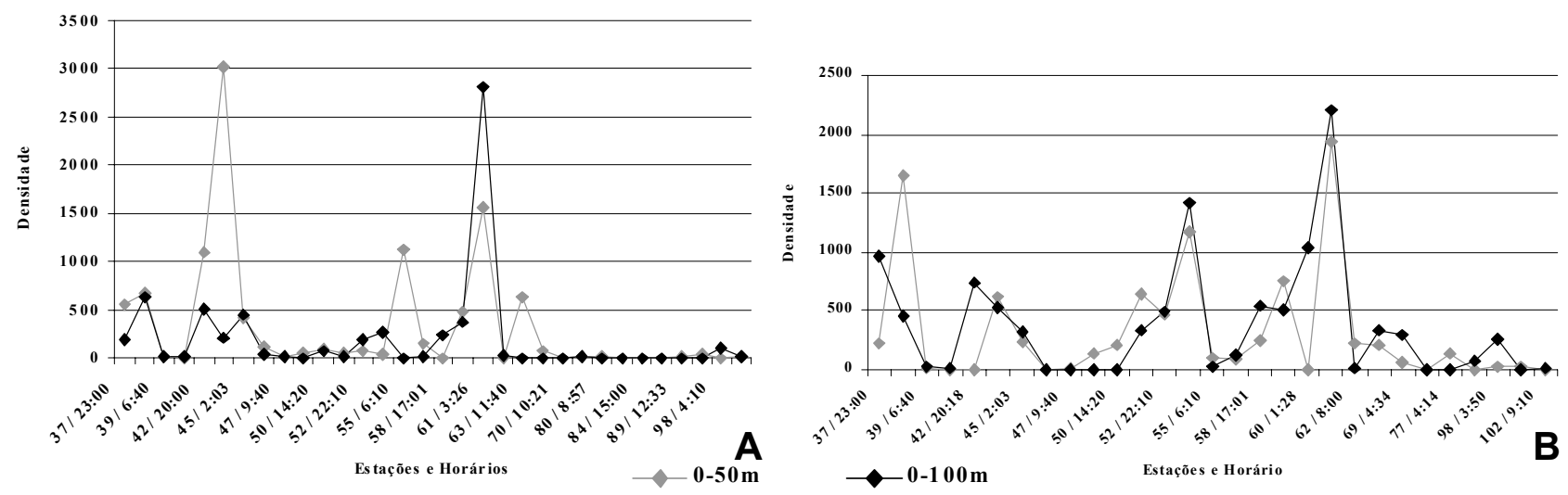

Figura 4. Densidade dos Pteropoda coletados na Cadeia Fernando de Noronha, com malha de (A) $300 \mu \mathrm{m}$ e (B) $500 \mu \mathrm{m}$ nos estratos de 0-50 e 0-100 m, durante a I Campanha do REVIZEE/NE, em 1995.

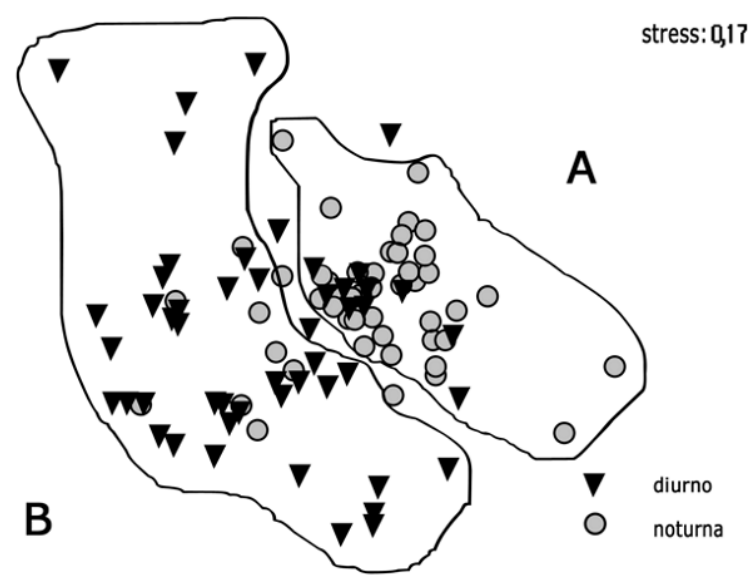

Figura 5. Representação gráfica em MDS da diferenças entre turnos na Cadeia Fernando de Noronha, durante a I Campanha do REVIZEE/NE, em 1995.

Fernando de Noronha foi aquela que apresentou para algumas espécies, os maiores valores de freqüência de ocorrência, corroborando os resultados obtidos por Boltovsкoy 1971, Magaldi 1974, Barth \& Oleiro 1968, Dadon 1984, Wormelle 1962, Wormuth 1981, Bé \& Gilmer 1977, entre outros.

Para a Cadeia Fernando de Noronha destacaram-se os Thecosomata (Euthecosomata): Limacina inflata, Creseis virgula, Cuvierina columnella e Cavolinia inflexa.

Limacina inflata constitui a espécie mais freqüente e dominante, apresentando maiores densidades nas coletas noturnas, tornando-se uma espécie comum nas amostras zooplanctônicas das áreas estudadas. Esta espécie foi considerada freqüente por Oliveira \& Larrazábal (2002) para o arquipélago São Pedro e São Paulo. Para o Atlântico Sul, Boltovskoy (1971), trabalhando no mar Argentino encontrou L. inflata como a segunda espécie mais freqüente, com maior quantidade de exemplares juvenis. MAgALDI (1974) também para a mesma área evidenciou que na maioria das estações, esta espécie foi considerada dominante e abundante, corroborando os nossos resultados. Em contrapartida, Resgalla \& MonTú (1994), considerando a sazonalidade de $L$. inflata em de dois cruzeiros oceanográficos, não encontraram para esta espécie alta densidade ou freqüência. Este resultado também fora obtido por BARTH \& OleIRO (1968) para as Águas Tropicais da Corrente do Brasil. DADON (1984) considera L. inflata o registro mais austral dos representantes dos Thecosomata sobre a plataforma continental, tendo sido ela, abundante. Neste aspecto, seus resultados coincidem com os obtidos para a Cadeia Fernando de Noronha. Segundo Wormelle (1962) L. inflata foi abundante e freqüente para o Atlântico Norte na Corrente da Flórida. Estes resultados se aproximam daqueles encontrados por Wormuth (1981), para o mar de Sargasso, onde $L$. inflata foi abundante e muito freqüente, tendo sido considerada uma espécie de águas quentes e cosmopolita, confirmado também por Bé \& Gilmer (1977), van der SPoel \& Boltovskoy (1981), van der Spoel \& Dadon (1999). Gasca \& SuárezMorales (1992) assinalaram L. inflata, como pouco comum para a Bahia de Ascension (México). SuÁrez-Morales \& GaSca (1992) relataram para o Golfo de Campeche (México), L. inflata como uma das espécies mais abundantes. Wells JR. (1973) registrou para a região de St. Ames (Barbados), L. inflata como a espécie mais comum e dominante. Para o mar da Arábia, Frontier (1963a) relacionou L. inflata como a quinta espécie de Pteropoda mais freqüente e a quarta mais abundante, relatando seu predomínio durante o período noturno, corroborando os nossos resultados. Frontier (1963b) para a ilha de Nosy-Bé (Madagascar), considerou L. inflata abundante e comum. HaAgensen (1976) estudando os Pteropoda do mar do Caribe afirmou ser $L$. inflata cosmopolita, predominantemente tropical e subtropical e a mais abundante entre as espécies, apresentando deslocamentos verticais, tanto de dia quanto à noite. Durante o período diurno uma menor concentração de indivíduos adultos foi observada na camada acima de $150 \mathrm{~m}$, entretanto, no período noturno a maior concentração foi observada acima de $100 \mathrm{~m}$. Os juvenis, na maioria das vezes, estiveram confinados acima de $90 \mathrm{~m}$, não apresentando diferenças entre os turnos amostrados. Próximo a Nosy-Bé (Madagascar), Frontier (1963b) encontrou 80 a 90\% de véliger de $L$. inflata na camada superior a $100 \mathrm{~m}$, tanto de dia quanto à noite. Na Corrente da Flórida Wormelle (1962) não encontrou diferenças entre dia e 
noite, apesar de observar uma ligeira tendência crescente à noite. Para a Cadeia Fernando de Noronha $L$. inflata apresentou maiores densidades durante a noite em ambos os estratos (050 e $0-100 \mathrm{~m}$ ), com diferença estatística significativa entre dia e noite. LALLi \& WellS JR. (1973) relataram pela primeira vez a proteção da cria pelo Thecosomata epipelágico L. inflata. Os adultos com conchas de diâmetro superior a $1,1 \mathrm{~mm}$ podem encubar embriões e precoces véliger na cavidade do manto. Através dessa adaptação na reprodução Bé \& GILmer (1977) atribuem o relativo sucesso dessa espécie e a seus altos índices de freqüência e abundância, a sua única forma de proteger sua prole na cavidade do manto durante o desenvolvimento.

Creseis virgula foi muito freqüente e freqüente em ambos os estratos, sobretudo em coletas diurnas, considerada abundante, com altas densidades na maioria das estações. Os mesmos resultados foram obtidos por OliveIRA \& LARRAZÁBAL (2002) para o arquipélago São Pedro e São Paulo. Com relação às densidades, Resgalla \& Montú (1994) e Barth \& Oleiro (1968) concordam com os resultados obtidos para a Plataforma Continental do Sul (Rio Grande do Sul) e Cabo Frio (Rio de Janeiro), respectivamente. Apontam C. virgula como uma espécie que apresenta baixas densidades. Entretanto, discordam com relação à freqüência e abundância relativa, onde para a Plataforma Continental Sul (Rio Grande do Sul) esta é freqüente e abundante no verão e para Cabo Frio (Rio de Janeiro) é esporádica e rara. Esses resultados apresentados para a costa Sul do Brasil são diferentes daqueles obtidos para a Cadeia Fernando de Noronha, fato este possivelmente devido a diferenças de temperatura entre as localidades estudadas, apesar de Bé \& GILMER (1977) assinalarem esta espécie como estenotérmica, podendo ser encontradas entre $7^{\circ}-31^{\circ} \mathrm{C}$. Para o Atlântico Norte, na Corrente da Flórida, Wormelle (1962) relata C. virgula como espécie abundante. De acordo com Wormuth (1981) em estudos sobre a distribuição vertical e sasonal dos Euthecosomata do Nordeste do mar de Sargasso, com relação à abundância e à freqüência de ocorrência, C. virgula mostrouse abundante, corroborando os resultados obtidos por WORMELLE (1962). Wells Jr. (1973) considerou esta espécie comum e também dominante para a região de St. Ames (Barbados). Frontier (1963a) para o mar da Arábia assinalou C. virgula, como a espécie mais freqüente e a mais abundante na maioria das amostras diurnas, concordando com os resultados obtidos para Fernando de Noronha. Observou também níveis de migração vertical diurna e noturna, apesar das maiores densidades terem sido encontradas em amostras coletadas no período diurno. FronTIER (1963b) para a ilha de Nosy-Bé considerou C. virgula abundante. HaAgENSEN (1976) encontrou duas formas desta espécie, sendo elas: Creseis virgula $f$. conica e Creseis virgula $f$. virgula, sendo a primeira, aproximadamente dezoito vezes mais abundante do que a segunda. Quanto à migração vertical, na Corrente da Flórida, WORMElle (1962) observou uma forte migração à superfície durante à noite, indicando ser $C$. virgula epipelágica, apresentando pequena migração vertical diurna. HaAgensen (1976) considerou similar a migração vertical, tanto para $C$. virgula $f$. conica como para C. virgula $f$. virgula. Ambas ocorreram acima de $80 \mathrm{~m}$, apresentando tendência à migração para superfície à noite. $\mathrm{Na}$ área de Fernando de Noronha, apesar de não terem sido consideradas as subespécies, C. virgula apresentou migração diurna, com maiores densidades obtendo valores estatisticamente significativos em ambos os estratos (0-50 e 0-100 m).

Cavolinia inflexa foi freqüente, abundante e rara para a área de Fernando de Noronha, dependendo da estação de coleta. De acordo com MAGALDI (1974) esta espécie obteve resultado semelhante no Atlântico Sul, onde sua distribuição esteve compreendida entre os paralelos $41^{\circ} \mathrm{N} \mathrm{e} 40^{\circ} \mathrm{S}$. Para o Atlântico Norte, Wormelle (1962) considerou C. inflexa como uma espécie rara. HaAgensen (1976) considerou que entre as espécies de Cavolinia, C. inflexa é a mais comum, ocorrendo em todo o Atlântico, tendo sido considerada abundante. Quanto à migração vertical desta espécie a maior densidade foi observada durante o dia. Wormelle (1962) evidencia migração diurna para a Corrente da Flórida. Para a área de Fernando de Noronha, esta espécie apresenta maior densidade no período noturno, em ambas as malhas coletoras, contradizendo os demais autores, confirmando aqueles obtido por Oliveira \& LARRAZÁbal (2002) para o arquipélago São Pedro e São Paulo.

Cuvierina columnella foi freqüente e pouco freqüente em ambas profundidades e na maioria das vezes, rara para Fernando de Noronha. Este resultado corrobora aqueles de MAGALDI (1974), que encontrou apenas dois exemplares em todas suas amostras. Para o Atlântico Norte, GaSCA \& SUÁrez-Morales (1992) encontraram apenas um exemplar, sendo considerada rara para a Bahia de la Ascension (México). Frontier (1963a) para o mar da Arábia encontrou apenas quatro indivíduos jovens, considerando-a uma espécie rara. Para o mar do Sargasso, WorMELLE (1962) relata que esta espécie ocorreu regularmente em suas amostras apesar de apresentar baixas densidades. HAaGENSEN (1976) considerou-a como espécie cosmopolita, abundante e tipicamente tropical e subtropical para o mar do Caribe. No que se refere à distribuição desta espécie na Corrente da Flórida, WORMELLE (1962) encontrou-a regularmente, apesar de evidenciar poucos exemplares nas amostras diurnas e uma maior quantidade à noite (cerca de 30 vezes mais do que aquelas coletadas durante o dia). Em Fernando de Noronha houve diferenças entre as densidades nos período diurno ou noturno, apesar dos testes estatísticos tendenciarem a uma maior densidade para o período noturno.

A espécie Limacina helicina (Phipps, 1774) tem sua distribuição geográfica evidenciada para correntes frias (BÉ \& Gilmer 1977, van Der Spoel \& Boltovskoy 1981). Segundo van Der Spoel \& Boltovskoy (1981), no que se refere às variações das correntes marinhas podem muitas vezes ocorrer influência na dispersão do plâncton de uma forma geral. Uma dessas influências relatadas pelos autores é que muitas espécies do Atlântico SulOcidental são euritérmicas em outras regiões de sua área de dispersão, sendo exemplo L. helicina, espécie rara para Fernando de Noronha, esta, caracterizada como região tropical. Oliveira \& Larrazábal (2002) estudando os Pteropoda do arquipélago São Pedro e São Paulo encontraram esta espécie, no que se refere à abundância relativa, como sendo pouco abundante a rara nas estações prospectadas, em ambos estratos e malhas coletoras, o que coincide com os resultados obtidos em Fernando de Noronha.

Os Pseudothecosomata foram esporádicos e raros, apresentando baixa densidade evalores muito baixos quanto à riqueza taxonômica para a área de Fernando de Noronha, representados pela espécie Corolla cupulla Rampal, 1996. Os indivíduos foram coletados em período noturno em sua maioria. HAAgensen (1976) relatou a presença de Pseudothecosomata para o mar do Caribe, apesar de nem todas as espécies estudadas para aquela área tenham sido consideradas raras.

Os Gymnosomata foram esporádicos e raros se comparados aos Thecosomata, apresentando baixas densidades. Entretanto, quanto à riqueza taxonômica apresentou um número de espécies superior aquele encontrado para os 
Pseudothecosomata e um pouco inferior aos Thecosomata. De acordo com Dadon \& Chauvin (1998) no mar da Argentina e Brasil - Confluência Malvinas, foram encontradas duas espécies: Spongiobranchaea autralis d'Orbigny, 1836 mais freqüente e abundante, e Clione antartica pouco abundante, ambas associadas à Corrente das Malvinas. Os Gymnosomata comportam espécies consideradas raras em amostras de zooplâncton (Dadon \& Chauvin 1998), o que corrobora os resultados obtidos em Fernando de Noronha. Os mesmos autores relatam a dificuldade de obtenção e identificação das espécies de Gymnosomata, às quais são coletadas através de cruzeiros oceanográficos, sendo as amostras fixadas em formaldeído, sem anestesiamento prévio dos espécimens. Esta metodologia dificulta a visualização dos exemplares que face ao fixador, contraem suas estruturas, impedindo a identificação. Esta conduta foi igualmente questionada no pretérito por Prouvot-Fol (1942). A maioria dos estudos sobre Gymnosomata tem sido realizados no Hemisfério Norte. No hemisfério Sul, somente listas taxomônicas podem ser encontradas na literatura, e as informações disponíveis, na realidade, são escassas (DADON \& CHAUvin 1998). MagAldi (1983), ao revisar 41 amostras obtidas em coletas no Sul do litoral brasileiro, reconheceu alguns exemplares de Pneumodermopsis paucidens (Boas, 1886), Gymnosomata pouco comum, de distribuição latitudinal descontínua. Considerou a presença dessa espécie, em igual proporção a outros Gymnosomata na comunidade zooplanctônica em águas brasileiras. Foi uma espécie pouco freqüente e pouco abundante entre os Pteropoda. É conhecido que os Gymnosomata não apresentam um padrão típico de migração vertical diário e, como conseqüência, é a causa das agregações noturnas de superfície. As maiores densidades de $P$. paucidens ocorreram no período diurno e as menores, no período noturno. $\mathrm{O}$ autor sugere que este fato esteja relacionado com a necessidade e disponibilidade de alimento, segundo o qual, sua concentração também se deveria ao comportamento alimentar da espécie. Pneumodermopsis paucidens é um ativo predador de Thecosomata se alimentando, sobretudo, de espécies do gênero Limacina e Creseis. Este fato explicaria o considerável efetivo de Limacina trochiformis e Creseis virgula junto a maior quantidade de $P$. paucidens em uma determinada estação. Por outro lado, a baixa densidade de $P$. paucidens em outra estação estaria diretamente relacionada a L. trochiformis e C. virgula, por haver poucos exemplares das espécies acima citadas nas amostras estudadas. MAGALDI (1983), relata a espécie $P$. paucidens para o Atlântico abaixo da linha do Equador, à sudeste do arquipélago de Fernando de Noronha $\left(4^{\circ} 4^{\prime} \mathrm{S}\right.$ e $\left.29^{\circ} 2^{\prime} \mathrm{W}\right)$, onde cita o primeiro registro desta espécie, com apenas um exemplar notificado. De acordo com o mesmo autor, os Gymnosomata se deslocam da superfície em direção às camadas mais profundas durante as horas de maior luminosidade. Esta afirmação corrobora os resultados obtidos para a Cadeia Fernando de Noronha, onde as maiores densidades ocorreram em coletas noturnas.

\section{CONCLUSÕES}

Há diferenças de freqüência de ocorrência entre os indivíduos das ordens Thecosomata e Gymnosomata da Cadeia Fernando de Noronha. Estas diferenças são evidentes entre os representantes da subordem Pseudothecosomata e os Gymnosomata.

Limacina inflata e Creseis virgula foram as espécies que mais se destacaram em freqüência de ocorrência, abundância relativa e densidade, seguidas por Cavolinia inflexa e Cuvierina columnella.

Limacina inflata apresentou maiores densidades em períodos noturnos e Creseis virgula em período diurno, em ambas as malhas coletoras.

De uma forma geral, as migrações verticais diárias, registraram maiores densidades de Pteropoda nas estações coletadas no período noturno, se comparada, às diurnas.

Com relação às densidades, não houve diferença significativa entre as profundidades, malhas coletoras e turnos, exceto para a malha de $300 \mu \mathrm{m}$, no estrato $0-100 \mathrm{~m}$ em ambos os turnos.

\section{AGRADECIMENTOS}

Ao Prof. José Carlos Nascimento de Barros, do Museu de Malacologia, Universidade Federal Rural de Pernambuco pela confirmação da determinação de algumas espécies. Ao Prof. Ralf Schwamborn pela confecção do abstract.

\section{REFERÊNCIAS BIBLIOGRÁFICAS}

Аввотт, R.T. 1974. American Seashells: the marine mollusca of the Atlantic and Pacific coast off North America. New York, Van Nostrand Reinhold, $2^{\text {nd }}$ ed., 633p.

BARTH, R. 1968. Ocorrência de indicadores biológicos em zona de ressurgência. Ministério da Marinha - Publicações do Instituto de Pesquisas da Marinha, Rio de Janeiro, 26:112.

Barth, R. \& T.A.P. Oleiro. 1968. Contribuição ao estudo dos moluscos planctônicos da região de Cabo Frio - RJ. Ministério da Marinha - Publicações do Instituto de Pesquisas da Marinha, Rio de Janeiro, 5: 1-8.

Bé, A.W.H. \& R.W.A. Gilmer. 1977. A zoogeographic and taxonomic review of Euthecosomatous Pteropoda, p. 733-808. In: A.T.S. RAMSAY (Ed). Oceanic micropaleontology. London, Academic Press, $1+\mathrm{I}+984 \mathrm{p}$.

Boltovskoy, D. 1971. Pteropodos thecosomados del Atlantico sudoccidental. Malacologia, Oxford, 11 (1): 121-140.

- 1981. Atlas del zooplancton del Atlántico sudoccidental y métodos de trabajo com el zooplancton marino. Mar del Plata, Publicación Especial del Instituto Nacional del Investigatión y Desarollos Pesquero, 936p.

Chen, C. \& A.W.A Bé. 1968. A seasonal distribuition of Euthecosomaous Pteropods in the surface water of five stations in the western north Atlantic. Bulletin of Marine Science of the Gulf and Caribbean, Amsterdam, 14 (2): 185-220.

Clarke, K.R. \& R.M. Warwick. 1994. Chance in marine communities: an approach to statistical analysis and interpretation. Bournemouth, Bourne Press, 128p.

DADON, J.R. 1982. Zooplanktonic recurrent groups (Pteropoda, Euphausiacea) southwestern Atlantic ocean. Physis, Buenos Aires, 41 (100):63-83.

- 1984. Distribuicion y abundancia de Pteropoda Thecosomata en el Atlantic sudoccidental. Physis, Buenos Aires, 42 (102): 25-38.

. 1990. Annual cycle of Limacina retroversa in Patagonia waters. American Malacological Bulletin, Arlington, 8 (1): 77-84.

. 1992. Abundancia and assemblages of planktonic Tunicata (Chordata) and Thecosomata (Mollusca) of south Brazil. Physis, Buenos Aires, 50 (118): 25-30. 
Dadon, J.R. \& N.H Magaldi. 1995. Mesoscale distribution of Thecosomata (Gastropoda) in the Brazil-Malvinas confluence compared with simultaneous satellite imagens of surface temperature. Iheringia, Série Zoologia, Porto Alegre, (78): 157-160.

Dadon, J.R. \& S.F. Chauvin. 1998. Distribuicion and abundance of Gymnosomata (Gastropoda: Opisthobranchia) in the southwest Atlantic. Journal of Molluscan Studies, London, 64, 345-354.

EHRHADT, J.P. \& G. SEGUIN. 1978. Le plankton: composition, écologie, pollution. Paris, Gauthier-Villares. 210p.

Frontier, S. 1963a. Zooplancton recolté en mer d'Arabie, Golfe Persique et Golfe d'Aden. II. Ptéropodes, Systématique et repartition. Travaux du Centre D'Océanographie et des Pêches de Nosy-Bé, Nosy-Bé, 6: 233-254.

-1963b. Heteropoda et ptéropodes recoltés dans le plankton de Nosy-Bé. Travaux du Centre D'Océanographie et des Pêches de Nosy-Bé, Nosy-Bé, 6: 213-227.

Gasca, R.S. \& E. SuÁrez-Morales. 1992. Pteropodos (Mollusca: Gastropoda; Thecosomata) de la bahia de la Ascension, reserva de la biosfera de Sian Ka'an, Quintana Roo, Mexico, p. 115-121. In: D. Navarro \& E. M. SuÁrez (Eds). Diversidad biológica en la reserva de la biosfera de Sian Ka'an Quintana Roo, Mexico. Quintana Rôo, II. CIQRO/ SEDESOL, 183p

Haagensen, D.A. 1976. Part II - Thecosomata, p. 551-772. In: H.B. Michel \& M. Foyo (Eds). Caribbean zooplankton. Miami, Office off Naval Research Departament of the Navy, 772p.

LALLI, C.M. 1970. Structure and function of the buccal apparatus of Clione limacina (Phipps) with a review of feeding in gymnosomatous pteropods. Journal of Experimental Marine Biology and Ecology, Amsterdam, 4: 101-118.

Lalli, C.M. \& F.E.L.B Wells Jr. 1973. Protection in Epipelagic Thecosomatous Pteropod, Spiratella ("Limacina") inflata (D'Orbigny). Bulletin of Marine Science of the Gulf Caribbean, Amsterdam, 23 (4): 933-941.

Lalli, C.M. \& T. Parsons. 1993. Biological oceanography: an introduction. Oxford, Pergamon Press, 301p.

Mabesoone, J.M. \& P.N. Coutinho. 1970. Litoral and shallow marine geology of Northeastern Brazil. Trabalhos do Instituto Oceanográfico Universidade Federal de Pernambuco, Recife, 12. 1-214.

Magaldi, N.H. 1974. Moluscos holoplanctonicos del Atlantico sudoccidental: I. Pteropodos euthecosomados colectados por el "Atlantis II" en marzo de 1971. Comunicaciones de la Sociedad Malacologica del Uruguay, Montivideo, 4 (27): $1-20$.

. 1981. Moluscos holoplanctonicos del Atlantico sudoccidental: IV. Thecosomados y gymnosomados de la campaña del "Hero" entre Puerto Deseado y Buenos Aires. Comunicaciones de la Sociedad Malacologica del Uruguay, Montivideo, 5 (41): 381-389.

- 1983. Moluscos holoplanctonicos del Atlantico sudoccidental: V. Hallazgo de Pneumodermopsis paucidens (Boas) en la bahia de Isla Grande y adyacencias (Brasil). Comunicaciones de la Sociedad Malacologica del Uruguay, Montivideo, 6 (44): 103-108.

MargalefF, R. 1974. Ecologia. Barcelona, Editora Omega, Casanova, 111p.
Oliveira, V.S. de \& M.E. De LarrazÁbal. 2002. Pteropoda (Gastropoda, Thecosomata e Gymnosomata) coligidos ao largo do arquipélago de São Pedro e São Paulo, costa Nordeste, Brasil. Revista Brasileira de Zoologia, Curitiba, 19 (Supl. 1): $215-227$.

Palma, J.J.C. 1984. Fisiografia da área oceânica. In: C. Schobbernhares (Ed). Geologia do Brasil. Brasília, Ministério das Minas e Energia, Departamento Nacional de Produção Mineral, 501p.

PéRÈs, J.M. 1976. Précis d'Océanographie biologique. Paris, Presses Universitaires de France, 247p.

Pruvot-Fol, A. 1942. Les gymnosomes. Dana Report $n^{\circ} 20$. Copenhagen: Carlsberg Foundation, 53p.

- 1954. Faune de France 58 Mollusques Opisthobranches. Paris, Office Central de Faunistique, 457p.

Resgalla, C.J. \& M. Montú. 1994. Distribuição espacial e temporal de pteropoda Euthecosomata (Mollusca Gastropoda) na plataforma do sul do Brasil. Atlântica, Rio Grande, 16 (1): 99-123.

Rios, E.C. 1974. Brazilian Marine Mollusks Iconography. Rio Grande do Sul, Editora Fundação Universidade do Rio Grande, XII+331p.

. 1985. Seashells of Brazil. Rio Grande Sul: Empresas Ipiranga. 328p.

. 1994. Seashells of Brazil. Rio Grande, Editora da Fundação Universidade do Rio Grande, $2^{\mathrm{a}}$ ed., 369p.

RUPPERT, E.E. \& D.R. BARNES. 1996. Zoologia dos Invertebrados. São Paulo, Rocca, $6^{\mathrm{a}}$ ed., 1029p.

Suárez-Morales, E.; R.S. Gasca. 1992. Pterópodos (Gastropoda: Thecosomata y Pseudothecosomata) de aguas superficiales (0-50 m) del sur del Golfo de México. Annales del Instituto de Ciencias Del Mar y Limnologia del Universidad Natural Autónoma, México, 19 (2): 201-209.

TesCH, J.J. 1946a. The thecosomatous pteropods: I. The Atlantic "Dana" Rep no 28. Copenhagen, Carlsberg Foundation, 82p.

- 1946b. The thecosomatous pteropods: I. The Atlantic “Dana" Rep no 28. Copenhagen, Carlsberg Foudation, $45 \mathrm{p}$.

VAN DER SPOEL, S. 1967. Euthecosomata a group with remarkable developmental stages (Gastropoda, Pteropoda). Gorinchem, J. Noorduijn en Zoon N.V., VIII+375p.

. 1972. Pteropoda Thecosomata. Conseil International pour l’Exploration de la Mer. Zooplankton, Paris, 8: 140-142.

- 1996. Pteropoda, p. 459-528. In: R. GaSCA \& E. SuÁreZ (Eds). Introducción al Estudio del Zooplancton Marino. México, El Colegio de la Frontera Sur (ECOSUR)/CONACYT, $771 \mathrm{p}$.

van der Spoel, S. \& D. Boltovskoy. 1981. Pteropoda, p. 493-533. In: D. Boltovsкoy (Ed.). Atlas del zooplancton del Atlántico sudoccidental y métodos de trabajo com el zooplancton mariño. Mar del Plata, Publicación Especial del Intituto Nacional de Investigatión y Desarrollo Pesquero, 936p.

van der Spoel, S. \& J.R. Dadon. 1999. Pteropoda, p. 640-706. In: D. Boltovskoy (Ed.). South Atlantic Zooplankton. Leiden, Backhuys Publishers, XVI+1706p.

Wells JR., F.E. 1973. Effects of mesh size on estimation os population densities of tropical Euthecosomatous Pteropods. Marine Biology, Berlin, 20 (4): 347-350.

Revista Brasileira de Zoologia 20 (2): 351-360, junho 2003 
WormelLe, R.L. 1962. A survey of the standing crop of plankton of the Florida Current. Bulletin of Marine Science of the Gulf \& Caribbean, Amsterdam, 12 (1): 95-136.
Wormuth, J.H. 1981. Vertical distributions and diel migrations of Euthecosomata in the northwest Sargasso Sea. Deep-Sea Research, Oxford, 28 (12): 1493-1515.

Recebido em 09.I.2003; aceito em 05.VI.2003.

Revista Brasileira de Zoologia 20 (2): 351-360, junho 2003 